\title{
Affordance theory can help understanding of individuals' use of online support communities
}

\author{
Neil S. Coulson
}

Division of Rehabilitation \& Ageing, School of Medicine, University of Nottingham, UK.

In the early days of the Internet limited interaction existed between websites and individual users and as a result individuals were largely confined to searching for and reading health-related information. Searching for health-related information remains commonplace (i.e. in 2016, 51\% searched for health information online in the UK, ONS, 2016). In addition, more recent technological advances have created forms of electronic communication which have encouraged participation, collaboration and information sharing between users (often referred to as 'Web 2.0'). One specific way in which this ability to interact with other users has manifest itself is through the development of online support communities (also known as 'online support groups'). Online support communities are a type of virtual community with a health-related focus, which provide an online environment where individuals can interact with other people who share common interests, experiences or concerns.

Whilst most online communities have been created to provide support to those affected by specific health conditions (e.g. HIV/AIDS), there are numerous communities which address other health-related topics, including behaviour change, parenting, caregiving and bereavement. Typically, online support communities are available 24 hours per day, 7 days per week and draw together a heterogeneous mixture of people, often from several countries and across multiple continents. Whilst online support communities can have several thousand registered members, the clear majority do not actively contribute content. In fact, it is widely acknowledged that only approximately $1 \%$ of users actively create new content with the remaining $99 \%$ being termed as 'lurkers' and prefer to read content rather than generate it (Katz, 1998). By far, the most common type of online support community is that which is underpinned by a discussion forum platform. Discussions are typically organised into 'threads' where one person starts a new conversation by posting an initial message (e.g. asking a question, describing a problem or talking about an experience that they want to share) and other members post replies to that message. Members might then post additional messages in response to these replies, thus building up a hierarchical thread of messages that stem from the original post.

Since the emergence of online support communities, researchers across a range of disciplines (e.g. psychology, sociology, communication) have taken a keen interest in their evolution, function and therapeutic potential. From a communication process-oriented perspective, Social Comparison Theory (Festinger, 1954); Strength of Weak Ties Theory, (Granovetter, 1973); and Social Information Processing Theory (Walther, 1992) have proven useful. In terms of health outcome-oriented perspectives, the Buffering Effect Model (Cobb, 1976) of social support has been the most widely applied and has yielded important insights into the potential health- 
related benefits of participation.

Whilst these theories have been helpful in advancing our knowledge concerning the process and outcome of online social support, there is a need to focus our attention more closely on the interaction between the individual community user and the specific functionality afforded by its underpinning platform and how this relates to health outcomes. Therefore, this editorial will propose that affordance theory may offer a helpful 'theoretical lens' through which to conceptualise this complex inter-relationship and it will illustrate from recent work how our understanding of online support communities can be advanced as well as identifying future research directions.

\section{Affordance Theory}

As psychologists, we have long been interested in the relationship between individuals and their environment and how their interaction shapes behaviour. The origins of affordance theory stem from perceptual and cognitive psychology and are based on how individuals perceive the objects in their environment, pertaining to both what the object is and what potential uses it affords (Gibson, 2015). The properties of an object will therefore contribute to its perceived affordance. Furthermore, the differing experiences, beliefs, and goals of an individual will lead to different perceived affordances, and so affordances are unique to individuals (Norman, 1988). The emphasis in the theory is on the interaction between the user and the object and its resulting outcomes. Therapeutic affordances can therefore be regarded as the "actionable possibilities" of the object as perceived by the user (Merolli, Gray and Martin-Sanchez, 2014), and the object in this instance is an online support community. Studying therapeutic affordances allows us to look at both the uses and effects of engagement with online support communities.

To date, there has been little attention devoted to understanding the therapeutic affordances conferred through engagement with online support communities. The work of Shoebotham and Coulson (2016) has attempted to address this and focussed on understanding the therapeutic affordances of online support communities for women living with endometriosis. In an asynchronous online interview with 69 participants, they described 4 therapeutic affordances related to engagement with online support communities, namely: (1) "connection," that is, the ability to connect in order to support each other, exchange advice, and to try to overcome feelings of loneliness; (2) "exploration," that is, the ability to look for information, learn, and improve their knowledge; (3) "narration," that is, the ability to share their experiences, as well as read about the experiences of others; and (4) "self-presentation," that is, the ability to manage how they present themselves online. Furthermore, the resulting outcomes, as reported by their respondents, were predominantly positive, such as reassurance and improved coping with the condition. However, it should be noted that this work also identified multiple negative aspects which included concerns surrounding the accuracy of information exchanged, arguments between community members, over-reliance on the community, becoming upset by negative experiences or good news items, and confidentiality of personal information.

Outside of the health domain, researchers have attempted to further develop the concept of therapeutic affordances by categorizing them into types based on their underlying function (Day and Lloyd, 2007). The findings of the Shoebotham and Coulson (2016) study can therefore be further interpreted using the 
framework proposed by Zhao, Liu, Tang and Zhu (2013), in which the perceived affordances are categorized into physical, cognitive, affective, and control functions. "Connection" may be regarded as an affective affordance, allowing individuals to find a community, seek support, and feel less alone. "Exploration" may best be considered as a cognitive affordance as it helps to facilitate the individual's thinking and learning. "Selfpresentation" may fall under control, as it allows individuals to control their environment, by maintaining their privacy for example. However, as Zhao et al., 2013 argues, affordances do not need to fall into these categories exclusively; for example, "narration" may be both affective, in providing reassurance, and cognitive as it allows individuals to learn from others' experiences.

A final point to note from the work of Shoebotham and Coulson (2016) is that only 4 therapeutic affordances were identified. In contrast, earlier work by Merolli et al., (2014) which considered social media use by individuals living with chronic pain identified a fifth therapeutic affordance, namely "adaptation", which relates to the way that social media allows users to adapt their self-management needs in relation to their health status or illness flare-ups. It may be that in the Shoebotham and Coulson (2016) study that evidence for "adaptation" was not found simply because no respondent commented upon it or because the affordance did not exist in this context. However, we should note that the data presented by Merolli et al., (2014) focussed on a broader array of social media platforms (e.g. blogs, video-sharing sites) as opposed to online support communities based solely on discussion forums. Therefore, we must be very careful to consider the specific social media platforms which underpin the online communities we are examining and pay attention to understanding the specific affordances that they may offer to individuals. In doing so, it may be the case that new affordances are identified and described and this is to be expected.

Whilst discussion forums remain the most common platform for online support communities, there has been an increase in the number of online communities developed using alternative social media platforms (e.g. social networking sites, such as Facebook; microblogging sites, such as Twitter and virtual worlds, such as Second Life). Future research should examine the therapeutic affordances conferred through specific social media platforms but health psychologists should also be mindful that individual social media platforms may rise or fall in popularity (e.g. Myspace). Consequently, it is also recommended that attention is given to understanding the overarching characteristics of the platforms which host online support communities (e.g. asynchronous versus synchronous; closed versus open). In doing so, insights may be obtained which extend beyond that of a specific platform. Researchers may also consider how individual factors (e.g. previous knowledge, motivation, attitudes) and/or illness-specific factors (e.g. current health status) relate to engagement with online support communities and how varying levels of engagement may relate to health outcomes. For example, it may be hypothesised that an individual who lacks specific digital literacy skills may choose to 'lurk' within an online community but may still benefit (i.e. through 'exploration') without actively generating content. Finally, the work of Shoebotham and Coulson (2016) reminds us that there may exist negative outcomes and these require careful and detailed consideration by health psychologists, not least so that strategies can be put in place to mitigate against potential problems.

\section{Conclusion}


This editorial has illustrated how future research concerning individuals' use of online support communities may be helped by considering their therapeutic affordances. By directing more attention to understanding the therapeutic affordances which exist within online support communities, we may be able to understand their potential contribution to individuals' self-management with greater clarity and focus.

\section{References}

Cobb, S. (1976). Social support as a moderator of life stress. Psychosomatic Medicine, 38(5), 300-314.

Day, D. and Lloyd, M.M. (2007). Affordances of online technologies: More than the properties of the technology. Australian Educational Computing, 22(2), 0816-9020

Festinger, L. (1954). A theory of social comparison processes. Human Relations, 7(2), 117-140.

Gibson, J. (2015). The ecological approach to visual perception. Psychology Press.

Granovetter, M. S. (1973). The strength of weak ties. American Journal of Sociology, 78(6), 1360-1380.

Katz, J. (1998). Luring the lurkers. Retrieved April 10, 2017, from

https://news.slashdot.org/story/98/12/28/1745252/luring-the-lurkers

Merolli, M., Gray, K. and Martin-Sanchez, F. (2014). Therapeutic affordances of social media: emergent themes from a global online survey of people with chronic pain. Journal of Medical Internet Research, 16(12), e284.

Norman, D. (1988). The psychology of everyday things. Basic Books.

Office for National Statistics (2016). Internet access - households and individuals: 2016. Retrieved from https://www.ons.gov.uk/peoplepopulationandcommunity/householdcharacteristics/homeinternetandsocialm ediausage/bulletins/internetaccesshouseholdsandindividuals/2016

Shoebotham, A. and Coulson, N.S. (2016). Therapeutic affordances of online support group use in women with endometriosis. Journal of Medical Internet Research, 18(5), e109.

Walther, J. B. (1992). Interpersonal effects in computer-mediated interaction: A relational perspective. Communication Research, 19(1), 52-90.

Wright, K.B. (2016). Communication in health-related online social support groups/communities: A review of research on predictors of participation, applications of social support theory, and health outcomes. Review of Communication Research, 4, 65-87.

Zhao, Y., Liu, J., Tang, J. and Zhu, Q. (2013). Conceptualizing perceived affordances in social media interaction design. In Aslib Proceedings, 65(3), 289-303. Emerald Group Publishing Limited. 